\title{
Efficient Neutron Production from a Novel Configuration of Deuterium Gas-Puff Z-Pinch
}

\author{
D. Klir, P. Kubes, K. Rezac, J. Cikhardt, J. Kravarik, and O. Sila \\ Czech Technical University in Prague, Faculty of Electrical Engineering, Technicka 2, 16627 Prague 6, Czech Republic \\ A. V. Shishlov, B. M. Kovalchuk, and N. A. Ratakhin \\ Institute of High Current Electronics SB RAS, 2/3 Akademichesky Avenue, Tomsk 634055, Russia
}

National Research Tomsk Polytechnic University, 30 Lenina Avenue, Tomsk 634050, Russia

V. A. Kokshenev, A. Yu. Labetsky, R. K. Cherdizov, F. I. Fursov, and N. E. Kurmaev

Institute of High Current Electronics SB RAS, 2/3 Akademichesky Avenida, Tomsk, 634055, Russia

G. N. Dudkin, B. A. Nechaev, and V. N. Padalko

National Research Tomsk Polytechnic University, 30 Lenina Avenida, Tomsk, 634050, Russia

H. Orcikova, and K. Turek

Nuclear Physics Institute, Academy of Sciences of Czech Republic, Na Truhlarce 39, 18086 Prague, Czech Republic

(Received 12 August 2013; published 3 March 2014)

\begin{abstract}
A novel configuration of a deuterium $z$ pinch has been used to generate fusion neutrons. Injecting an outer hollow cylindrical plasma shell around an inner deuterium gas puff, neutron yields from DD reactions reached $Y_{n}=(2.9 \pm 0.3) \times 10^{12}$ at $700 \mathrm{~ns}$ implosion time and 2.7 MA current. Such a neutron yield means a tenfold increase in comparison with previous deuterium gas puff experiments at the same current generator. The increase of beam-target yields was obtained by a larger amount of current assembled on the $z$-pinch axis, and subsequently by higher induced voltage and higher energies of deuterons. A stack of CR-39 track detectors on the $z$-pinch axis showed hydrogen ions up to $38 \mathrm{MeV}$. Maximum neutron energies of 15 and $22 \mathrm{MeV}$ were observed by radial and axial time-of-flight detectors, respectively. The number of DD neutrons per one joule of stored plasma energy approached $5 \times 10^{7}$. This implies that deuterium gas puff $z$ pinches belong to the most efficient plasma-based sources of DD neutrons.
\end{abstract}

DOI: 10.1103/PhysRevLett.112.095001

PACS numbers: 52.58.Lq, 52.70.Nc

$Z$ pinches are known as efficient and powerful generators of soft $\mathrm{x}$-ray radiation [1]. At present, there has been also a growing interest in neutron production in $z$ pinches. There are three main reasons for this renewed attention: (i) the MAGLIF project [2,3], (ii) the high DD neutron yield of $4 \times 10^{13}$ in a 15 MA deuterium gas puff on the $Z$ machine [4-7], and (iii) a need for pulsed sources of fast neutrons. Small repetitive portable neutron sources could be useful in radiation material science, radiobiology, nuclear medicine, cargo inspection, and illicit material detection, whereas large devices could be effective for the recycling of nuclear waste and for a fusion-fission hybrid reactor.

Attempts have been made to obtain neutron numbers sufficient for these applications. For instance, laser driven ions were used to produce neutron beams [8]. It is also reasonable to research deuterium $z$ pinches as compact sources of fast neutrons since they had produced a large number of DD fusion neutrons already in the 1950s. To achieve high neutron yields, various configurations based on the $z$-pinch effect have been suggested and tested from that time on. The most promising configuration seemed to be a dense plasma focus (DPF) with a deuterium gas filling. The beam-target mechanism in a DPF produced $10^{11} \mathrm{DD}$ neutrons/shot at a stored energy of $100 \mathrm{~kJ}$ [9]. Plasma foci demonstrated the dependence of a neutron yield per length on a current as $Y_{n} / l \propto I^{4}$ up to $I=1 \mathrm{MA}$ [10]. Unfortunately, this favorable scaling law was not extended above 1 MA. The "saturation" of neutron yields between $10^{11}$ and $10^{12}$ was observed on the mega-ampere plasma foci in Frascati, Limeil, Moscow, Stuttgart, and Warsaw (e.g., Refs. $[10,11])$. The lack of scaling beyond $10^{12}$ was one of the most important arguments for shutting down the largest plasma focus facilities in the 1980s. In the 1990s, neutron yields above $10^{12}$, namely up to $2.8 \times 10^{12}$, were reached with a deuterium gas puff $z$ pinch on Saturn, however, at much higher currents of 8 MA [12].

In order to investigate neutron production mechanisms and to understand better the process of the saturation of beam-target yields, we decided to carry out $z$-pinch experiments on a MA current level. Neutron measurements at this current level are also very important considering the development of rep-rated mega-ampere current generators. In this Letter, we present highly efficient neutron production from a novel configuration of a deuterium gas puff $z$ pinch. When an inner deuterium gas puff was surrounded by an outer hollow cylindrical plasma shell injected from 
plasma guns, neutron yields above $10^{12}$ were achieved at 3 MA currents. Comparing the obtained results with the previous gas puff experiments at the same current but without using plasma guns [13], the neutron yield was increased by a factor of 10 . Furthermore, hydrogen ions up to $38 \mathrm{MeV}$ and $\mathrm{DD}$ neutrons up to $22 \mathrm{MeV}$ were observed for the first time in $z$ pinches. A high neutron production efficiency of $5 \times 10^{7}$ DD neutrons per one joule of stored plasma energy resulted from the generation of high energy deuterons and probably also from their magnetization inside plasmas.

Setup.-The experiments were carried out on the GIT-12 generator at the IHCE in Tomsk [14]. GIT-12 capacitors were charged to $50 \mathrm{kV}$. A schematic diagram of our experimental setup is depicted in Fig. 1. An anode-cathode gap varied between 23 and $27 \mathrm{~mm}$ in most of the shots. Cathode and anode mesh transparency was $\approx 70 \%$. A total linear mass of deuterium gas in single or double shell gas puffs was usually between 80 and $120 \mu \mathrm{g} / \mathrm{cm}$. Outer and inner nozzle diameters were 80 and $30 \mathrm{~mm}$, respectively. In contrast to the previous experiments with triple shell gas puffs [13], the plasma shell of ionized hydrogen and carbon ions was injected from 48 plasma guns at the diameter of $350 \mathrm{~mm}$ (see Ref. [15] for more details). When the plasma guns were triggered 1.7-1.9 $\mu$ s before the onset of the main current, the linear mass of the plasma shell was about $5 \mu \mathrm{g} / \mathrm{cm}$.

The dynamics of the gas puff $z$ pinch was observed by a four-frame time-gated x-ray pinhole camera, an optical streak camera, four $B$-dot probes, x-ray diodes, photoconducting detectors, a fast bolometer, and voltage and current probes [13]. The emphasis was put on neutron diagnostics. Neutron energies were measured by four radial and one axial neutron time-of-flight (TOF) detectors at the distances between 170 and $2579 \mathrm{~cm}$ from the source. The TOF detectors were shielded against the bremsstrahlung radiation by up to $20 \mathrm{~cm}$ of lead. Neutron yields were calculated from a silver activation counter, from TasTrak CR-39 detectors filtered by $4 \mathrm{~mm}$ of lead [16], and from five bubble detectors (BD-PNDs) [17] placed at different distances and angles. In order to subtract the contribution of

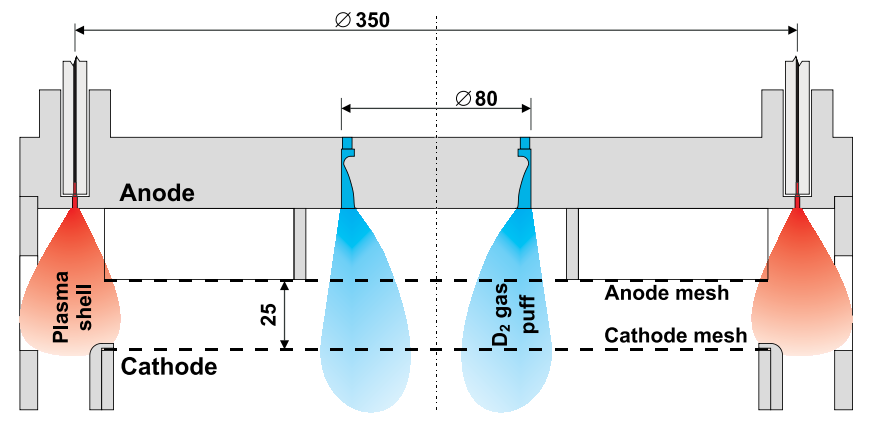

FIG. 1 (color online). Schematic diagram of the experimental setup with a single shell gas puff. scattered neutrons, great attention was paid to the absolute calibration of BD-PNDs and CR-39 detectors by an AmBe neutron source in situ. One BDS-10000 superheated fluid detector [18] was placed in the radial direction to measure $>10 \mathrm{MeV}$ neutrons. Finally, to detect fast ions, two stacks of $1.1 \mathrm{~mm}$ thick CR-39 detectors were placed axially and radially 19 and $58 \mathrm{~cm}$ from the source, respectively.

Experiments.-During two experimental campaigns, 37 shots were performed with the plasma shell on the deuterium gas puff. Using the configuration shown in Fig. 1, a gas density profile was relatively smooth. For the total linear mass density of $\approx 100 \mu \mathrm{g} / \mathrm{cm}$, the implosion from a $350 \mathrm{~mm}$ diameter lasted $700 \pm 50 \mathrm{~ns}$ and seemed to be stable up to a $5 \mathrm{~mm}$ radius. Figure 2 shows a typical example of soft $\mathrm{x}$-ray images of the final stage of the implosion when the current rose to 2.7 MA. The implosion driven by the $\mathbf{J} \times \mathbf{B}$ force reached a radial velocity of $4.5 \times 10^{5} \mathrm{~m} / \mathrm{s}$. During stagnation, $m=0$ instabilities became more pronounced. When a disruption of necks occurred, high energy ( $>2 \mathrm{MeV}$ ) bremsstrahlung radiation and a main neutron pulse were observed. The peak neutron yield from DD reactions reached $Y_{n}=(2.9 \pm 0.3) \times 10^{12}$. The reproducibility of shots with $2 \times 10^{12}$ yields was approximately $50 \%$. Calculating with the neutron emission time of $20 \mathrm{~ns}$, we obtain the production rate of $10^{20} \mathrm{n} / \mathrm{s}$. The neutron yield per unit length exceeded $10^{12} \mathrm{n} / \mathrm{cm}$ with double as well as with single shell gas puffs. Shots of $\approx 2 \times 10^{12}$ neutron yields were confirmed in two independent experimental campaigns within the time span of 5 months.

In order to achieve neutron yields above $10^{12}$, we had to fulfill three necessary conditions (if any of these were not met, the neutron yield was on the order of $10^{11}$ ): First, we used an optimal gas puff mass of about $100 \mu \mathrm{g} / \mathrm{cm}$. This optimal value was explained by a tradeoff between the energy of fast deuterons and the number of fast and target deuterons $[13,19]$. Second, it was necessary to set the time delay between valve opening and triggering of the generator below $350 \mu \mathrm{s}$. For the longer delays, there was a significant gas spread at large distances, which made the current concentration onto the axis more difficult [13]. Finally, high neutron yields were achieved only when an outer shell was formed by plasmas injected from the plasma

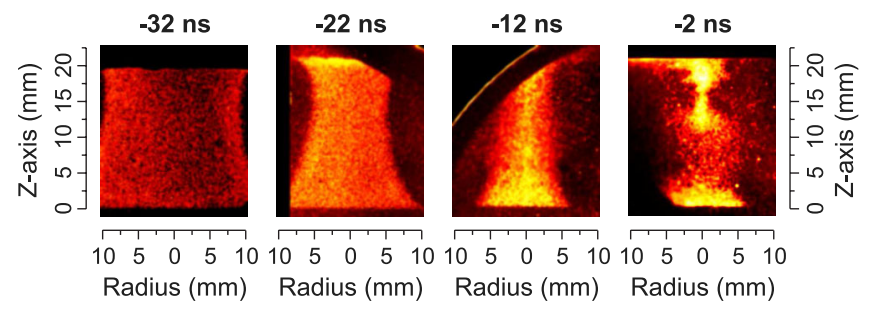

FIG. 2 (color online). Gated soft x-ray images of the deuterium gas puff $z$ pinch. The time $t=0$ corresponds to the sharp onset of $>2 \mathrm{MeV}$ bremsstrahlung radiation and the start of main neutron emission. 
guns. The idea behind using the plasma shell was to form a uniformly conducting layer at a large initial radius. A stable implosion of a uniform layer is needed to bring currentcarrying plasmas close to the axis. An outer plasma shell is expected to eliminate most of the problems associated with gas puffs (low ionization degree, inhomogeneous breakdown, etc.) and to minimize mass left behind the main implosion. In the case of an outer gas shell, the mass left behind is probably more significant. Later in the implosion, the residual mass is likely to become highly conductive and a relatively large current-carrying region may be formed. Especially at stagnation, high induced voltage can cause restrike of current in the residual mass. This way, the current assembled on the axis at disruption can be reduced.

In order to verify these presumptions we placed several $B$-dot probes just above the cathode mesh at different distances from the $z$-pinch axis. When an outer gas shell (from Ref. [13]) was replaced by the plasma shell, the $B$-dot probe at a $3 \mathrm{~cm}$ radius showed an increase of the current at stagnation from $\approx 1.0$ to $\approx 1.6 \mathrm{MA}$ and a compression of its rise time (10\%-90\%) from 50 to $30 \mathrm{~ns}$. In both cases, the total current of $2.7 \mathrm{MA}$ at stagnation remained the same. Furthermore, by using the plasma guns, the implosion at soft x-ray images seemed to be more stable and the plasma impedance at the disruption grew from 0.14 up to $0.35 \Omega$. The energy input into plasmas up to the end of neutron emission $W_{\text {input }}=\frac{1}{2} \int \dot{L} I^{2} d t$ and the peak power $V I$ were increased from 40-50 kJ to 60-70 kJ and from 1 to 2 TW, respectively. These results suggest that restrikes were mitigated, which is important for induction of high voltages and acceleration of deuterons to high energies.

The increase of deuteron energies by using plasma guns can be illustrated by a substantial difference between

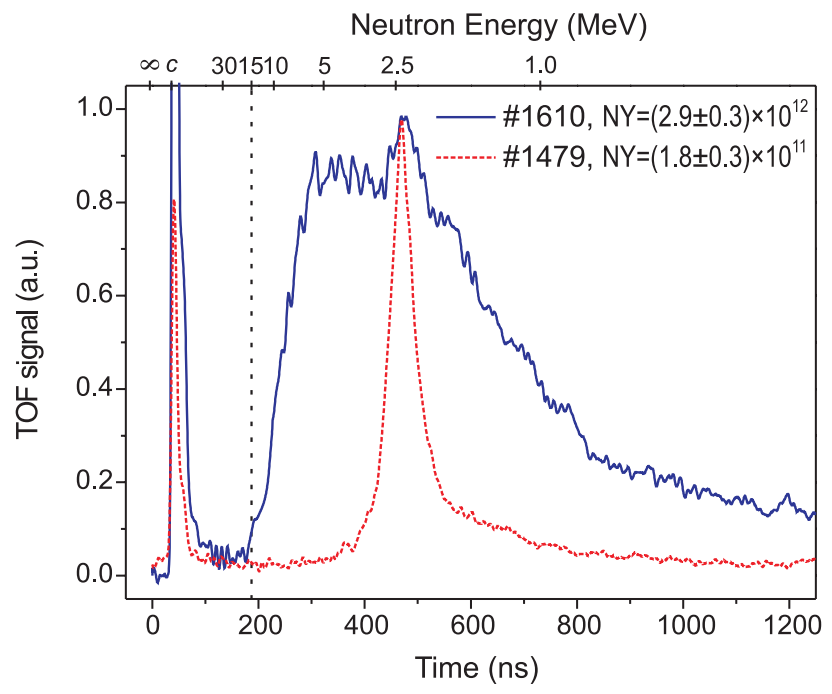

FIG. 3 (color online). Neutron TOF signals at $10.13 \mathrm{~m}$ in a high yield shot (\#1610, plasma shell on single shell gas puff) and a low yield shot (\#1479, triple shell gas puff without plasma guns [13]) for 2.7 MA current at stagnation, $100 \mu \mathrm{g} / \mathrm{cm}$ linear mass of $D_{2}$ gas. neutron spectra in low yield $\left(2 \times 10^{11}\right)$ and high yield $\left(2 \times 10^{12}\right)$ shots. Figure 3 shows radial neutron time-offlight (nTOF) signals measured at $10.13 \mathrm{~m}$. In the high yield shots, the maximum neutron energies in the radial direction were $15.5 \pm 0.5 \mathrm{MeV}$. The number of $>10 \mathrm{MeV}$ neutrons in the radial direction reached the value of $(3 \pm 1) \times 10^{9} \mathrm{n} / \mathrm{sr}$. Most of the neutrons originated from $\mathrm{D}(d, n)^{3} \mathrm{He}$ reactions but a certain number of neutrons could be produced also by secondary $D(t, n)^{4} \mathrm{He}$ reactions, deuteron breakup $(d, n p)$, deuteron electrodisintegration $d\left(e, e^{\prime} n\right) p$, and other endothermic reactions. In any case, it follows from Fig. 3 that the high yield regime was achieved by a large number of deuterons with higher $(\approx \mathrm{MeV})$ energies.

Neutron energies even higher than $15 \mathrm{MeV}$ were measured by the axial nTOF detector at $475 \mathrm{~cm}$. Downstream, i.e., in the axial direction towards the cathode, the peak neutron energy reached $22 \pm 1 \mathrm{MeV}$. We believe that this is a record value for DD neutrons in $z$-pinch plasmas. Assuming stationary target deuterons, such a high energy means that a large number of deuterons were moving with $20 \mathrm{MeV}$ kinetic energy in the axial direction. In order to verify high energy deuterons on the axis directly, we placed the stack of five CR-39 track detectors below the cathode mesh. Figure 4 shows deuteron- and/or proton-induced tracks in the CR-39 detectors. Proton-induced tracks had to be taken into consideration because of the composition of plasmas injected by the plasma guns into the discharge. As shown in Fig. 4, the CR-39 detectors confirmed the presence of high energy ions on the axis: the first CR-39 layer at $19 \mathrm{~cm}$ was saturated, implying more than $10^{10}$ of $>15 \mathrm{MeV}$ hydrogen ions per steradian. For the energies $E$ above $15 \mathrm{MeV}$, the number of hydrogen ions $N(E)$ decreased rapidly according to a power law distribution as $d N / d E \propto E^{-5}$. Nevertheless, there were still protons or deuterons with kinetic energies up to 38 or $51 \mathrm{MeV}$, respectively, since the number of tracks behind $4.25 \mathrm{~mm}$ of aluminum and $3.3 \mathrm{~mm}$ of CR-39 was still significantly above "neutron background." The observed energies were about 5 times greater than the maximum deuteron energy observed in previous experiments $[13,20]$. The peak ion beam energy of $38 \mathrm{MeV}$ is significantly in excess of the inferred voltage across the pinch, and much larger than the applied pinch voltages for the largest pulsed power facilities $[4,5,7,12]$.

Efficient production of beam-target neutrons.-The results mentioned above indicate that most of the neutrons in our experiment were beam target in origin. Even though the beam-target mechanism in $z$ pinches has been studied for decades, it is still a source of controversy, which has not been resolved [21]. One of the problems is the scaling of neutron yields with a current. In the case of the beam-target mechanism, the $I^{4}$ scaling might be degraded at a certain current. In fact, the saturation of neutron yields was observed in MA DPFs in the 1980s. From this point of 


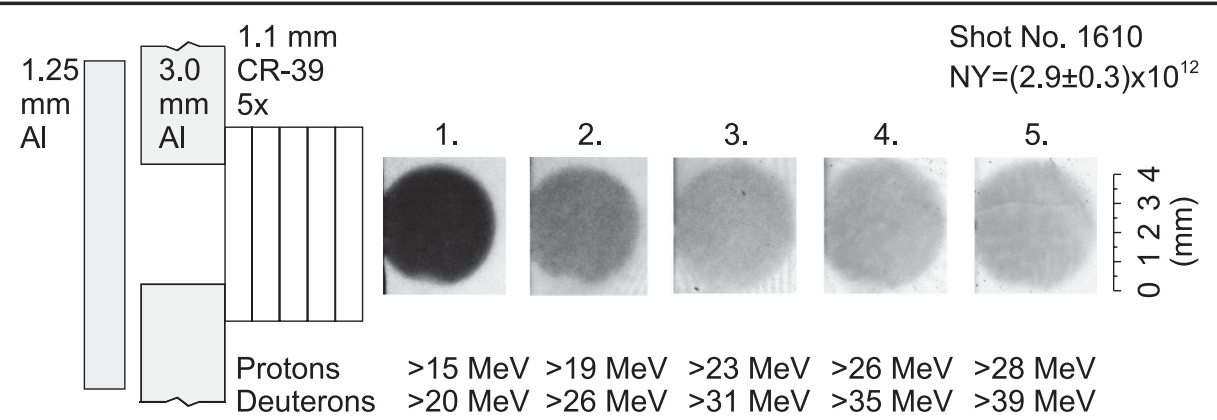

FIG. 4. Proton- and/or deuteron-induced tracks in the stack of five CR-39 detectors (each of 1.1 mm thickness) placed on the axis at $19 \mathrm{~cm}$ from the $z$ pinch and etched for 2 hours in $30 \% \mathrm{KOH}$ at $70{ }^{\circ} \mathrm{C}$. A $1.2 \mathrm{~mm}$ thick aluminum foil was used as an absorber. Circular footprints were caused by a $4 \mathrm{~mm}$ diameter hole inside a $3 \mathrm{~mm}$ thick aluminum case before the CR-39 stack.

view, the yield above $10^{12}$ can be considered as a significant achievement. Taking into account the scaling law in Krishnan's review paper [10], the yield of $2 \times 10^{12}$ at 2.7 MA fits to the $I^{4}$ scaling law observed in dense plasma foci. As for published $z$-pinch and dense plasma focus experiments, DD neutron yields higher than $2.9 \times 10^{12}$ were achieved only on $Z$, however, at very high currents of about $15 \mathrm{MA}$. Also, according to fully kinetic simulations, the yields we have achieved are more than 1 order of magnitude higher than predictions for $3 \mathrm{MA}$ and are expected to be achieved rather on 8 MA generators [22]. Moreover, these kinetic simulations [22] are capable of explaining broad radial neutron spectra in a MA $z$ pinch. On the other hand, however, the production of $>10 \mathrm{MeV}$ neutrons and deuterons has not been anticipated. All these results suggest that more theoretical and experimental work is needed to understand ion acceleration mechanisms and the beam-target production of neutrons in $z$ pinches. In this respect, simultaneous measurements of ions and neutrons could provide valuable data for $z$-pinch physics. For instance, in our experiment, a broad width of side-on neutron spectra implies that the neutrons were not produced by a monoenergetic, collimated beam of accelerated deuterons. In contrast, the observed neutron spectra can be explained by a suprathermal distribution of deuterons with the high energy tail $f\left(E_{d}\right) \propto E_{d}^{-k}$. Following the procedure described by Knapp et al. [23], a good fit of the radial neutron spectra in high yield shots was obtained for the isotropic composite distribution of ions with a power law index $k$ of $1.8 \pm 0.2$ and an ion temperature of $\leq 2 \mathrm{keV}$. If the high energy tail is known, it is possible to calculate the total energy of deuterons accelerated to fusion energies $W_{>20 \mathrm{keV}}$ from the neutron yield $Y_{n}$, ion density $n_{d}$, and ion path length $l$ as

$$
W_{>20 \mathrm{keV}}=\frac{Y_{n}\left\langle E_{d}\right\rangle_{>20 \mathrm{keV}}}{n_{d} l\left\langle\sigma\left(E_{d}\right)\right\rangle_{>20 \mathrm{keV}}}
$$

Here $\left\langle E_{d}\right\rangle$ and $\left\langle\sigma\left(E_{d}\right)\right\rangle$ stand for the ion energy and the $D(d, n)^{3} \mathrm{He}$ cross section averaged over the high energy tail, respectively. For $k=1.8$, the ratio between $\left\langle E_{d}\right\rangle_{>20 \mathrm{keV}}$ and $\left\langle\sigma\left(E_{d}\right)\right\rangle_{>20 \mathrm{keV}}$ equals $14 \mathrm{keV} / \mathrm{mbarn}$. During the main neutron emission, the average plasma radius $R$ estimated from pinhole and streak images was $4 \mathrm{~mm}$. Because the line density was $N=$ $3 \times 10^{19}$ deuterons/cm, the ion density reached $n_{d}=N /$ $\pi R^{2}=6 \times 10^{19} \mathrm{~cm}^{-3}$. If we assume the path length of fast deuterons $l \approx 2 \mathrm{~cm}$, the neutron yield $Y_{n} \doteq 3 \times 10^{12}$ leads to the total energy $W_{>20 \mathrm{keV}}$ of $60 \mathrm{~kJ}$. Such a value is similar to the total energy input into the plasma. However, this does not seem realistic considering that the tail formed only a fraction $(\approx 0.1 \%)$ of all deuterons. The discrepancy can be overcome if the path length $l$ is chosen longer than $2 \mathrm{~cm}$. A longer trajectory could be the result of ion magnetization.

The magnetization of deuterons can be evaluated by the ratio between the Larmor $r_{\text {Larmor }}$ and the pinch radius $R$. For deuteron kinetic energy $E_{d}$, mass $m_{d}$, charge $e$, and the magnetic field $B$ produced by the cylindrical current $I$, the ratio $r_{\text {Larmor }} / R$ is given by

$$
\frac{r_{\mathrm{Larmor}}}{R}=\frac{m_{d} \sqrt{2 E_{d} / m_{d}}}{e B R}=\frac{2 \pi \sqrt{2 E_{d} m_{d}}}{e \mu_{0} I} \approx \frac{\sqrt{E_{d}[\mathrm{MeV}]}}{I[\mathrm{MA}]} .
$$

Assuming $E_{d}=10 \mathrm{MeV}$ and $I=2 \mathrm{MA}$, we obtain the ratio $r_{\text {Larmor }} / R \approx 1.6$. This means that deuterons below $10 \mathrm{MeV}$ could be strongly influenced by magnetic fields generated by a 2-3 MA current assembled on the axis. We do not have any indication that $>15 \mathrm{MeV}$ deuterons were magnetized in our experiment. Nevertheless, the magnetization of deuterons below $10 \mathrm{MeV}$ can be deduced by comparing signals from neutron and ion detectors. The radial flux of 4-14 MeV neutrons above $5 \times 10^{10} \mathrm{n} / \mathrm{sr}$ implied that a large number of $1-10 \mathrm{MeV}$ deuterons was moving in the radial direction. Since the radial CR-39 detectors recorded only $3 \times 10^{9}$ of $>1 \mathrm{MeV}$ deuterons/sr and since the conversion efficiency of deuterons into neutrons is low, deuterons seemed to be trapped inside plasmas. Last but not least, the magnetization of ion beams follows from a low anisotropy of the neutron flux of about $30 \%$ and from plasma dimensions. On the one hand, the 
axial and radial neutron fluxes were similar. Therefore, "effective" path lengths of ions in the axial and radial directions were also comparable. On the other hand, however, the length to diameter ratio of the plasma column was about 4. If we put both statements together, we conclude that some of the fast ions were magnetized. This means that the high neutron yields were caused not only by higher energies of deuterons but perhaps also by their magnetization inside plasmas.

As mentioned above, the CR-39 detectors showed only a few ions in the radial direction. There were, however, a lot of fast ions escaping the $z$ pinch along the axis. Therefore utilizing these ions offers the real possibility of a further increase in neutron yields. Indeed, when a deuterated polyethylene foil of $15 \mathrm{~mm}$ diameter and $0.9 \mathrm{~mm}$ thickness was placed onto the axis $3 \mathrm{~mm}$ below the cathode mesh, the peak neutron yield reached $(3.7 \pm 0.4) \times 10^{12}$. Since the energy input into the plasma was $65 \pm 5 \mathrm{~kJ}$, the number of neutrons per one joule of stored plasma energy reached $5 \times 10^{7}$. A higher efficiency of DD neutron production on the order of $10^{9} \mathrm{n} / \mathrm{J}$ was obtained in the largest tokamaks, such as JET and JT-60U, at a much higher input energy. Taking into consideration also a high $(\approx 10 \%)$ conversion efficiency of stored electrical energy into z-pinch plasmas, deuterium gas puff $z$ pinches belong to the plasma-based sources of DD neutrons with the highest wall-plug efficiency (e.g., Ref. [4]).

To conclude, we have shown that the novel configuration of a mega-ampere deuterium $z$ pinch is able to produce a large number of $\gtrsim 1 \mathrm{MeV}$ deuterium ions, some of which may be magnetized. The magnetization of deuterons appears to be favorable for efficient production of fusion neutrons. The advantage of employing neutrons in $z$-pinch applications is that fast neutrons can penetrate through chamber walls whereas troublesome debris produced by a $z$ pinch remains within an experimental chamber. Thus, it seems natural to research $z$ pinches not only as sources of $\mathrm{x}$ rays but also as efficient plasma-based sources of neutrons.

The authors are grateful to Dr. A. L. Velikovich for many useful discussions. This research was supported by the GACR (Grant No. P205/12/0454), by the MSMT (Project Nos. LH 13283 and LG 13029), by the IAEA (Grant No. RC 17088), by the MES RF (Project No. 2.1704.2011), by the IHCE SB RAS State Task No. 13.1.4, by the RFBR (Project No. 13-08-00479-a), and by the Program for Basic Research of the Presidium of RAS "Fundamental problems of pulsed high-current electronics." *daniel.klir@fel.cvut.cz

[1] T. Sanford, G. Allshouse, B. Marder et al., Phys. Rev. Lett. 775063 (1996).

[2] D. B. Sinars, S. A. Slutz, M. C. Herrmann et al., Phys. Rev. Lett. 105, 185001 (2010).

[3] S. A. Slutz and R. A. Vesey, Phys. Rev. Lett. 108, 025003 (2012).

[4] C. A. Coverdale, C. Deeney, A. L. Velikovich et al., Phys. Plasmas 14, 022706 (2007).

[5] C. A. Coverdale, C. Deeney, A. L. Velikovich et al., Phys. Plasmas 14, 056309 (2007).

[6] A. L. Velikovich, R. W. Clark, J. Davis et al., Phys. Plasmas 14, 022701 (2007).

[7] D. R. Welch, D. V. Rose, R. E. Clark, C. B. Mostrom, W. A. Stygar, and R. J. Leeper, Phys. Rev. Lett. 103, 255002 (2009).

[8] M. Roth, D. Jung, K. Falk et al., Phys. Rev. Lett. 110 044802 (2013).

[9] A. Bernard, H. Bruzzone, P. Choi et al., J. Mosc. Phys. Soc. 8, 93 (1998).

[10] M. Krishnan, IEEE Trans. Plasma Sci. 40, 3189 (2012).

[11] H. Herold, A. Jerzykiewicz, M. Sadowski, and H. Schmidt, Nucl. Fusion 29, 1255 (1989).

[12] R. B. Spielman, G. T. Baldwin, G. Cooper et al., "D-D Fusion Experiments Using Fast Z Pinches," Sandia National Laboratories Report No. SAND98-0705, 1998 (unpublished).

[13] D. Klir, A. V. Shishlov, V. A. Kokshenev et al., Plasma Phys. Controlled Fusion 55, 085012 (2013).

[14] S. P. Bugaev, A. M. Volkov, A. A. Kim et al., Russ. Phys. J. 40, 1154 (1997).

[15] V. A. Kokshenev, A. Yu. Labetsky, B. M. Kovalchuk et al., "Multi-Shell Plasma Flow Switch Experiments on the GIT12 Generator," in Proceedings of 14th Symposium on High Current Electronics, Tomsk Russia, 2006 (Institute of High Current Electronics, Tomsk, 2006), pp. 272-275.

[16] J. A. Frenje, C. K. Li, F. H. Seguin et al., Rev. Sci. Instrum. 73, 2597 (2002).

[17] H. Ing, R. A. Noulty, and T. D. McLean, Radiation Measurements 27, 1 (1997).

[18] H. Ing, Radiation Measurements 33, 275 (2001).

[19] D. Klir, V. A. Kokshenev, P. Kubes, A. Yu. Labetsky, M. Paduch, K. Rezac, and A. V. Shishlov, IEEE Trans. Plasma Sci. 41, 3129 (2013).

[20] R. L. Gullickson and H. L. Sahlin, J. Appl. Phys. 49, 1099 (1978).

[21] M. G. Haines, Plasma Phys. Controlled Fusion 53093001 (2011).

[22] D. R. Welch, D. V. Rose, C. Thoma, R. E. Clark, C. B. Mostrom, W. A. Stygar, and R. J. Leeper, Phys. Plasmas 17, 072702 (2010).

[23] P. F. Knapp, D. B. Sinars, and K. D. Hahn, Phys. Plasmas 20, 062701 (2013). 